\title{
Monitoring of hygiene in institutional kitchens in Belgium
}

Duthoo E. ${ }^{1}$, Krings S. ${ }^{2}$, Daube G. ${ }^{2}$, Taminiau B. ${ }^{2}$, Heyndrickx M. ${ }^{1}$, De Reu K. ${ }^{1}$

${ }^{1}$ Flanders Research Institute for Agricultural, Fisheries and Food (ILVO), Technology and Food Science Unit, Brusselsesteenweg 370, 9090 Melle, Belgium ${ }^{2}$ University of Liege, FARAH Center, Department of Food Science - Microbiology, Quartier Vallée 2 (B43b), Avenue de Cureghem 10, B-4000 Liège, Belgium

\section{INTRODUCTION}

Good hygiene in institutional kitchens is essential for the prevention of foodborne illness. Respecting the basic rules of hygiene is the basis to guarantee safety in this sector where many steps are manual and different equipment is used.

To have a better understanding of the hygiene situation and problems, critical food contact surfaces and hand contact in institutional kitchens were monitored with classic microbiology.

These results will be compared In a later stage with metagenomics of the same samples (results not shown).

\section{MATERIALS AND METHODS}

\section{0 kitchens}

- 10 hospitals

- 10 schools

- 10 retirement homes

10 daycares

589 surfaces sampled
Sampling method

- New pair of gloves before every surface

- Swab using moistened cotton pad

- Repeated swab with dry cotton pad

- Checklist of 36 questions regards to food handler's practices and hygiene conditions

\section{Parameters}

- Total aerobic count

- Enterobacteriaceae

- Bacillus cereus

- Escherichia coli

- Staphylococcus aureus

Surfaces: hands, cutting knives, cutting boards, ladles, workbenches, trays and extras (whisks, strainers, blenders or cutting machines) Always sampled before and after cleaning

Also sinks and aprons

\section{RESULTS}

Table 1: Descriptive values for total mesophilic aerobic

microorganisms counts on swab samples of surfaces before cleaning.

\begin{tabular}{|ccc|}
\hline Surface & Positive (\%) & log CFU/20 cm ${ }^{2}$ \\
\hline Hands & $40(100)$ & $3.4 \pm 1.0$ \\
Cutting Knife & $37(95)$ & $2.3-4.0-5.0$ \\
Cutting Board & $31(100)$ & $2.5-3.8-5.2$ \\
Ladle & $33(87)$ & $2.3 \pm 1.3$ \\
Worktable & $39(100)$ & $2.0-3.3-4.3$ \\
Tray & $30(94)$ & $1.8-2.2-2.8$ \\
Extra & $31(89)$ & $1.9-3.0-5.2$ \\
Sink & $40(100)$ & $4.0 \pm 1.5$ \\
Apron & $30(97)$ & $2.4 \pm 0.9$ \\
\hline Table $2:$ Descriptive & values for total & mesophilic aerobic \\
microorganisms counts on swab samples of surfaces after cleaning. \\
\hline Surface & Positive (\%) log CFU/20 cm ${ }^{2}$ \\
\hline Hands & $40(100)$ & $2.9 \pm 1.1$ \\
Cutting Knife & $33(83)$ & $2.7 \pm 1.5$ \\
Cutting Board & $38(100)$ & $1.7-2.1-3.6$ \\
Ladle & $36(97)$ & $1.4-2.2-3.0$ \\
Worktable & $36(92)$ & $3.1 \pm 1.6$ \\
Tray & $35(100)$ & $2.3 \pm 1.0$ \\
Extra & $38(78)$ & $2.6 \pm 1.3$ \\
\hline
\end{tabular}

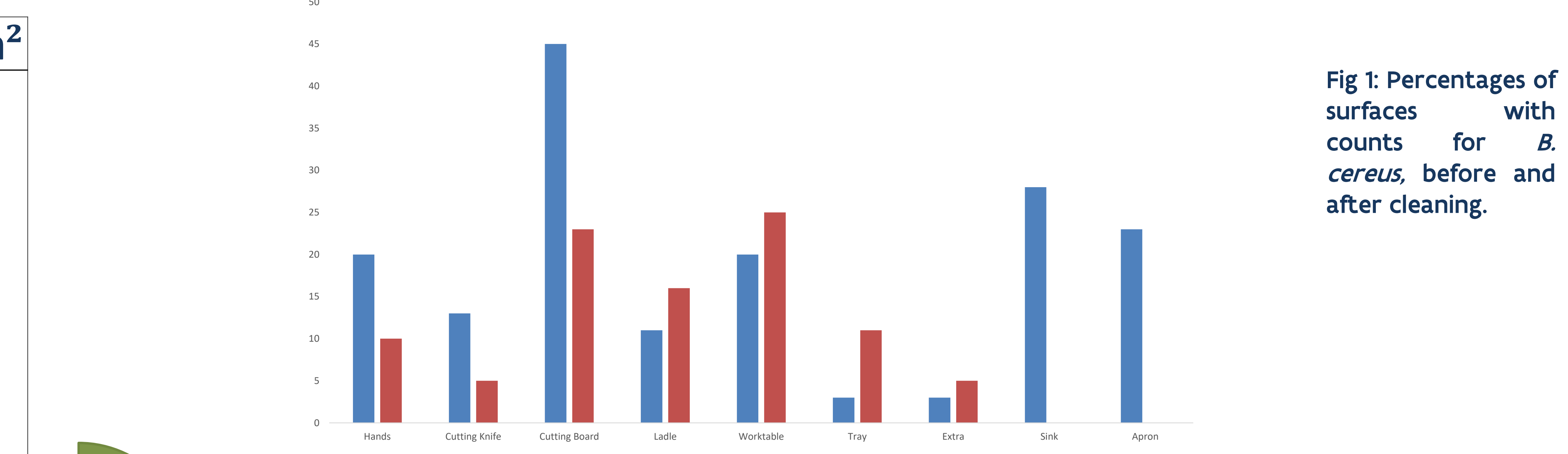

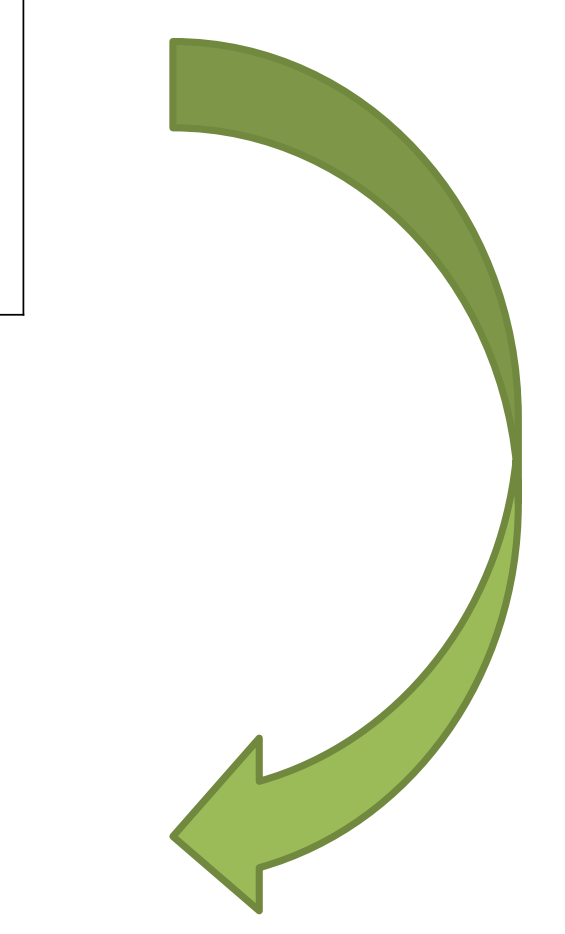

Mean and
standard deviation are given for normally distributed counts. First quartile, median and third quartile are given distributed counts.

\section{CONCLUSIONS}

Remarkably, used cutting knives and -boards had highest counts for total mesophilic aerobic microorganisms and were comparable with sinks; highest reduction was also obtained after cleaning.

Cleaned worktables had highest counts for total mesophilic aerobic microorganisms of cleaned surfaces. Also lowest reduction after cleaning.

Ladles, worktables, trays and extra's, more samples tested positive for B. cereus after cleaning than before cleaning. For ladles and the extra's, same situation for S. aureus.

$45 \%$ of worktables and $33 \%$ of cutting boards still test positive for Enterobacteriaceae after cleaning.

Hygiene score lowest in day cares.
Table 3: Hygiene scoring (based on checklist) of kitchens per sector (maximum score $\mathbf{3} 36$ ).

\begin{tabular}{|ccc|}
\hline Sector & N & Score \\
\hline Hospitals & 10 & $28.8-30.4-32.0$ \\
Schools & 10 & $29.3-31.7-34.2$ \\
Retirement homes & 10 & $30.1-30.5-32.4$ \\
Day cares & 10 & $24.4-26.8-28.5$ \\
\hline
\end{tabular}

